cases, three (Cases 1, 3, 5) had previously failed to respond satisfactorily to E.C.T. It will be evident from this series that synhexyl is as effective in the milder forms of dysoxia as in the purely neurotic-depressive states.

As regards the mechanism of improvement, it should be pointed out that synhexyl does not effect a permanent "cure" in the same way as electro-anoxia does in typical dysoxic depressions. The effect lasts only during the period of administration of the drug; it is therefore a substitution therapy, like insulin treatment in diabetes or liver extract in pernicious anaemia. The action would seem to be a combination of stimulation and depression, resulting in a general raising of the anhedonic threshold. The principal site of action of the drug is almost certainly the thalamic centres and their cortical connexions, as is the case with morphine and other powerful central analgesic drugs.

The beneficial effects of synhexyl do not appear to be adversely affected by chronicity, concomitant organic disease, or the presence of an organic brain lesion, whether pathologically or surgically induced. There is evidence, however, that drugs of this class are ineffective in the acute dysoxic depressions, and cannot be regarded as substitutes for anoxic therapy in this form of metabolic brain disease.

\section{General Conclusions}

The results of these preliminary trials would suggest that we have in this class of compounds a promising therapeutic agent for the treatment of the chronic and intractable depressive states. Synhexyl, the most active of this class, has the advantages of low toxicity, minimum of sideeffects, ease of administration, and chemical stability. Its use is not contraindicated by the presence of coexisting organic disease, and it is suitable for out-patient practice. Its use does not interfere with other therapeutic measures, such as occupational therapy and psychotherapy. It is free from the risks and disadvantages of the more drastic forms of treatment, and might replace those methods for the milder depressions of later life where for any reason the more drastic procedures are contraindicated.

Its main drawbacks at present are its insoluble nature, slow and uncertain action, and comparatively weak analgesic effect, so that it is relatively ineffective in the severer forms of sensory thalamic dysfunction syndrome. Experiments are at present in progress with the object of producing a water-soluble form with a higher degree of analgesic activity.

\section{Summary}

The syndrome of thalamic dysfunction and the principles of its treatment with euphorigenic drugs are described.

The properties and pharmacology of synhexyl, a new drug of the synthetic tetrahydrocannabinol class, are examined.

The results of therapeutic trials with the drug in a series of 50 depressive patients are given.

My acknowledgments are due to the following: The Research Department of Roche Products Ltd. and Dr. W. S. Maclay, of the Board of Control, for kindly providing supplies of synhexyl ; Dr. W. A. Bain, of Leeds University, for kindly making up the material for administration; Prof. A. R. Todd, of Cambridge University, for kindly supplying purified cannabis extracts for clinical trial; and Mrs. E. Bathurst, psychologist, Warlingham Park Hospital, for kindly undertaking psychometric tests on the patients tested with the drug.

\section{BIBLIOGRAPHY}

Adams, R. (1941-2). Harvey Lectures, 37, 168.

- and others (1941). J. Amer. chem. Soc., 63, 1971

- Baker, B. R., and Wearn, R. B. (1940). Ibid., 62, 2204

Allentuck, S., and Bowman, K. M. (1942). Amer. J. Psychiat., 99, 248 .

Ghosh, R., Todd, A. R., and Wilkinson, S. (1940). J. chem. Soc., pp. 1121,1893

Himmeisbach, C. K. (1944). Sth. med. J., Nashville. 37, 26.

1 and Andrews, H. L. (1943). J. Pharmacol., 77, 17.

Russell, P. B., and others (1941). j. chem. Soc., p. 826.

Stockings, G. T. (1944). J. ment. Sci., 90, 772.

\section{TYPE I TRIPLE CARDIAC RHYTHM IN NORMAL HEARTS}

\section{A STUDY OF 1,360 NAVAL RECRUITS AND PERSONNEL}

BY

\author{
P. J. O'MEARA, M.B., Ch.B. \\ Surgeon Lieutenant-Commander, R.N.
}

Triple cardiac rhythm is defined by Evans (1943) as " the cadence produced when three sounds recur in successive cardiac cycles." He then classifies such rhythm into types I, II, and III, which correspond respectively to the protodiastolic, presystolic, and systolic forms of "gallop" rhythm of Potain (1856-1900). The term "gallop" is not used in Evans's classification because it is usually only when tachycardia is also present that a cadence somewhat inadequately described as resembling the gallop or canter of a horse is found to be present.

Type I triple rhythm results from the presence of a third heart sound occurring shortly after the normal second. Thayer $(1908,1909)$ called attention to the fact that the third heart sound, described by Gibson (1907), Hirschfelder (1907), and Einthoven (1907), and mentioned even earlier by Obrastzow (1905), could often be heard in healthy people ; he found it in $65 \%$ of 231 consecutive individuals below the age of 40 . Subsequently Gallavardin (1912) said that he heard it occasionally in young individuals. Bridgman (1915) reported it in 13 out of 16 normal boys aged 12 to 15 and found it in phonocardiograms of all. Obrastzow and Gubergritz (1919) heard the sound in 90 to $93 \%$ of normal men ; Steinberg (1925) in $95 \%$ of boys between the ages of 4 and 14 ; and Melik-Gülnasarian (1932) in $57 \%$ of his cases, especially in young men between 20 and 25 years of age. Bramwell (1943) encountered it in $18.8 \%$ of 835 recruits referred to him by medical boards of the Ministry of Labour and National Service. Among the 228 individuals in this group below 20 years of age it was present in $43 \%$. None of these recruits had a cardiac lesion.

The purpose of the present purely clinical investigation is to emphasize the frequency of type I triple rhythm in healthy males and to study its features. That its common incidence is not appreciated enough nor its features sufficiently well known is evident from the number of times it gives rise to a diagnosis of mitral stenosisespecially when accompanied, as it may well be, by a mitral systolic murmur and perhaps a split second sound in the pulmonary area. A history of rheumatic fever in such an individual is then accepted as the final link in the diagnosis. As Evans (1943) remarks, "A general acceptance of the fact that this form of triple rhythm is so common in young subjects is overdue." That it is far from rare in the notso-young requires emphasis also.

\section{Method and Material}

The subjects of this survey consisted at first of 1,000 consecutive healthy male adults between 17 and 30 years of age inclusive who underwent routine examination by me for a variety of purposes. Later 360 men above the age of 30 were examined. All these individuals were considered healthy on the grounds that they presented no symptoms or physical signs of any disease whatsoever ; nor did they give any history of any cardiovascular disease. Some of the older men showed palpable thickening of the radial arteries commensurate with their age, but any who had blood pressures above 160 systolic or 90 diastolic were excluded. 
The following routine was adopted in the examination of the heart in every one of these subjects : examination (i) in the erect position, (ii) in the recumbent position, (iii) lying on the left side (left lateral position), and (iv) in the left lateral position after sitting up quickly from the recumbent position six times. Before proceeding to stage three -that is, with the subject on his back-the pulse rate and blood pressure were recorded. In the few cases in which time permitted the performance of exercise tolerance tests, or in which these were compulsory, as in Fleet Air Arm candidates, the information so obtained gave the impression of bearing no significant relation to the occurrence of triple rhythm.

Only those examples of type I triple rhythm were recorded which were evident with the subject erect or lying down, since the examination of the heart does not usually go beyond this stage unless the doctor has some reason to proceed further before eliminating the possibility of a cardiac lesion.

\section{Incidence of a Third Heart Sound}

Table I shows the frequency with which a third sound was recorded among 1,360 Naval recruits and personnel.

TABLE I.-Incidence of a Third Heart Sound Among 1,360 Healthy Males

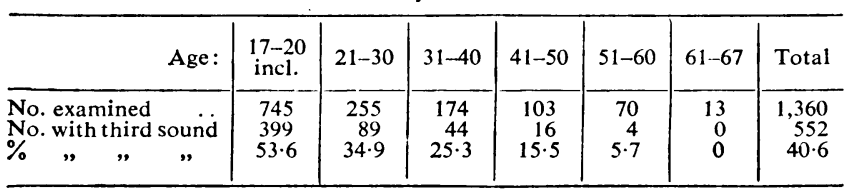

As already described, the examination of the 1,000 aged 17 to 30 inclusive was completed before that of the older group was begun. Now, although I had been impressed by the frequency of a third sound before beginning the present investigation, it was found that by listening intently for it in case after case the sensitivity of the ear was increased to a remarkable degree ; this may be seen from a study of Table II, which gives the incidence of the sound among the last 400 of the 1,000 males under 31 years of age.

TABLE II.-The Third Sound in the Last 400 Males Below 31 Years of Age

\begin{tabular}{|c|c|c|c|c|}
\hline & Age: & $17-20$ & $21-30$ & Total \\
\hline $\begin{array}{l}\text { No. examined .. } \\
\text { No. with third sound } \\
\%, ", \|, \quad \ldots\end{array}$ & $\ldots$ & $\begin{array}{c}325 \\
231 \\
71 \cdot 08\end{array}$ & $\begin{array}{c}75 \\
37 \\
49 \cdot 3\end{array}$ & $\begin{array}{l}400 \\
268 \\
67 \cdot 0\end{array}$ \\
\hline
\end{tabular}

It is considered that this figure of $67 \%$ forms a more accurate estimate of the incidence of the third sound among males of this age group than that obtained from Table I $(399+89=488$ or $48.8 \%)$.

\section{Some Features of the Third Heart Sound}

Characteristics.-These have been aptly described by various authors as "short and dull, a sort of thud" (Potain, 1856-1900), "dull shock rather than sound" (Thayer, 1908, 1909). Potain further likened it to the recoil of a drum or the rebound of. a hammer on an anvil. As a rule the third sound is of less intensity than either of the other two. However, Wolferth and Margolies (1933) remarked that occasionally it might be the loudest of the three, and this was found to be not unusual among the younger members of the present series. Frequently in the present series the third sound was palpable at the apex beat; sometimes it was visible also. In no case could it be more easily felt than heard even when a pronounced protodiastolic gallop rhythm was present. "Gallop" rhythm is usually described as being more easily felt than heard, but it is possible that this is generally true only of the presystolic variety which results from the presence of a fourth sound.

Site of Maximum Intensity.-The third sound is usually described as being heard best at or near the apex beat. Evans (1943), on the other hand, lays stress on the fact that in normal individuals he found it most audible in the fourth intercostal space, half-way between the nipple line and the left border of the sternum ; in other words, a little internal to the mitral area. In every case in which I identified a third heart sound it was loudest at the site of the apex beat. When faint it was present at the apex beat only; when more prominent it could be heard with diminishing intensity for a distance of about $2 \mathrm{in}$. $(5 \mathrm{~cm}$.) outside the nipple area-in rarer cases even as far out as the anterior axillary line. As a rule it was heard furthest from the apex beat internally, along the left sternal border or along a line passing from the apex beat towards the pulmonary area ; occasionally it was faintly audible in the pulmonary area itself. In a rather nervous young man of 23 it could be heard as high as the left sterno-clavicular joint, as far out as the mid-axilla, and as far to the right as the right sternal border. In another youth, aged 19, it was audible in the mid-axilla and beyond the right sternal border. In both, however, it was maximal at the apex beat.

Influence of Age upon the Third Sound.-Previous writers have remarked upon the decreasing incidence of this sound with advancing age (Thayer, 1908, 1909 ; Evans, $1943,1945)$, and this is clearly illustrated among the present subjects. Evans (1945) states definitely that it is never found in a healthy heart after the age of 40 . However, the figures in Table $I$ do not bear this out. Certainly in the sixth decade the sound is unusual, while beyond this age it is possible that it never occurs in the absence of disease. Thayer (1909) suggested that this increasing rarity of the sound with age might be explained by the comparative thinness and elasticity of the chest wall in the young and the absence of intervening lung. After the age of 40 , and especially after 50 , all the heart sounds become increasingly difficult to hear and their characters alter. They become shorter, more distant, and less resonant; often the first sound can scarcely be heard at all. It is not surprising, therefore, that the third sound, which is usually of much less intensity than either of the other two in healthy people, should be so difficult to hear in older subjects.

Influence of Posture.-In only two of the 1,360 subjects could a third sound be heard at stage (i) of the examination - that is, with the individual erect. The ages of these two were 17 and 19, and both were of poor physique ; in each case the sound was much louder with the subject recumbent. In some cases, examined after rising from recumbency, a third sound continued, but only so long as the pulse rate produced by the act of rising was maintained. Evans (1943) made similar observations on 125 normal hearts presenting a third sound, though in his series the sound persisted in the erect position in 13. However, all the patients examined by Evans had been sufficiently problematical to require reference to him for further opinion and were therefore, in a sense, selected.

Influence of Respiration.-In the majority of the present series I found the third sound most audible in normal expirations; with forced expiration it often disappeared. Sometimes, usually among the older members of the series, it was most easily heard at about mid-inspiration. Unless very prominent it was absent at the height of normal inspiration, and in almost every case it disappeared on forced inspiration.

Influence of the Pulse Rate.-The lowest pulse rate among those with a third heart sound was 48 ; the highest 
132 ; the average 80 . The last compared with an average of 76 in those without the sound. Bramwell (1943) also noticed no significant difference between the pulse rate of those with and those without the sound. Thayer (1909) considered the former to have a somewhat slower rate than the latter. $\mathrm{He}$ also gained the impression that the sound was more pronounced in subjects who had just lain down than in those who had been longer recumbent. I frequently noticed that a third heart sound would disappear as the examination of the recumbent patient continued. More often, however, a third sound would appear for the first time after the subject had been lying down for a minute or two. Again, the production of tachycardia by means of exercise, while generally increasing the intensity of the sound, would in some cases decrease it-occasionally causing the sound to disappear. All this suggests that in each individual there is an optimum pulse rate, varying from case to case, at which the sound is best heard. Probably the force of the heartbeats is just as important a factor in the production of the sound.

Influence of Blood Pressure.-The average systolic blood pressure among those presenting a third sound was 135 and the average diastolic 70 ; among those without the sound it was 130 and 70 respectively. Hence the presence or absence of a third sound appears to be independent of the blood pressure.

Means of Intensifying the Third Sound.-Thayer (1909) described various methods by which the third sound could be intensified. Raising the arms or legs, pressure on the abdomen, turning the subject on to the left side, and the production of tachycardia were suggestions he made. I found it possible to carry out only the last two as a routine in my cases, and there was no doubt that in the vast majority the sound was exaggerated in the left lateral position. The effects of tachycardia have been noted above.

\section{The Significance of Type I Triple Rhythm}

It is evident that an audible third sound at the apex beat, with the subject recumbent, is a common finding in health below middle age. It is not surprising, therefore, that it should happen to be present sometimes in a heart that is diseased, and its occurrence in such circumstances can surely be of no help to the clinician either in making a diagnosis or in forming a prognosis. On the other hand, it appears that a third sound, clearly and constantly heard in the erect position, is a rarity in a healthy heart and calls for the most careful investigation and consideration before being dismissed as of no significance. It may be that it usually is of some significance, and the louder the sound the more likely it is to be so. Even in the recumbent position a very loud third sound-that is, one as loud as or louder than either of the other two-appears to be unusual in men over 40 , though not so uncommon in the young.

This influence of position as a means of distinction between a third sound in a healthy heart and in one that is - diseased has already been remarked upon by Evans (1943). $\mathrm{He}$ also considered that in the presence of cardiac disease a third sound was maximal in the mitral area itself or external to it, whereas in the normal heart it was maximal internal to the mitral area. Since the third sound in the subjects reported in the present paper was invariably maximal at the apex beat $I$ cannot agree that its presence in the mitral area has any significance.

White (1928) stated that " gallop" rhythm, in which term he included protodiastolic gallop resulting from a third sound, was always a sign of considerable gravity in civil life ; and that during the 1914-18 war, when soldiers with effort syndrome were gathered together, a combination of pronounced tachycardia and a loud third heart sound occasioned a minimization of the importance of gallop rhythm widely at variance with the appreciation of its significance in civilian practice. In the most recent edition of his book on heart disease (White, 1944) he asserts that protodiastolic "gallop" is more serious than presystolic. Now, among the present series of healthy males it was observed that what was usually required for a so-called protodiastolic gallop to be heard was an individual with both a third sound and a pulse rate of over 100 . Furthermore, such a rhythm could be produced in many other subjects with a third sound by simply inducing a tachycardia by exercise. There was no reason to suppose that any of these people were sufferers from effort syndrome or that they might be so in the future. In other words, it appears that a protodiastolic "gallop" is not always a sign of gravity. On the contrary, it is heard quite frequently in normal hearts, especially among the young. When present in the recumbent position alone in a patient with heart disease it is probable that what is of significance is not the "gallop" rhythm but the tachycardia which produces it.

In certain of these men a type I "gallop" was present with a pulse rate of 90 ; in one it was heard with a rate of only 84 . No doubt this production of a so-called "gallop" cadence with the slower pulse rate occurs when there is a longer interval than usual between the second and third sounds.

\section{The Distinction between Type I Triple Rhythm and a Split Second Sound}

It will be seen that no use is made of the term "reduplication," or, more correctly, "duplication." A heart sound is never duplicated. Either it is split into its normal components or an extra sound is present. The application of the term "reduplication" to both these phenomena, as usually happens, leads only to confusion.

Distinctions between a split second sound and an audible third sound are as follows: (i) the former is nearly always most obvious in the pulmonary area, while the latter has been found to be always maximal in the lower part of the praecordium-in my experience at the apex beat itself, whether the heart be normal or abnormal ; (ii) splitting of the second sound is maximal on full expiration and, as I have found, on full inspiration; at both of which times, but especially the latter, a third sound is usually absent; but, what is most important, the cadence produced by a third heart sound is so characteristic that it should be impossible for anyone familiar with it to mistake the typical "lup-dupty" rhythm for a splitting of the second. Subjects in whom an audible third sound extends sufficiently high or a split sound sufficiently low show the two phenomena occurring in the same situation, the third sound clearly following the second component of the split sound. The cadence thus produced may be described as "lup-brupty."

Finally it is hoped that the time may not be far off when the standard textbooks devote more space to this and other forms of triple rhythm so that students may know what to expect and what not to expect from a normal heart.

\section{Summary}

The frequency of type I triple rhythm due to a third heart sound among 1,360 healthy Naval recruits and personnel is emphasized. The characteristics of the third sound, the site of maximum intensity, and the influence upon it of age, posture, respiration, and pulse rate are noted.

The usual insignificance of this form of triple rhythm, as judged from these findings, is noted.

Comparison is briefly made between this form of triple rhythm and a split second sound. 
I am grateful to the Medical Director-General of the Royal Navy for permission to publish this paper.

\section{REFERENCES}

Bramwell, C. (1943). Brit. Heart J., 5, 24.

Bridgman, E. W. (1915). Heart, 6, 41

Einthoven, W. (1907). Pflügers Arch. ges. Physiol, 120, 31

Evans, W. (1943). Brit. Heart J., 5, 205.

- (1945). Practitioner, 155, 198.

Galiavardin, L. (1912). Arch. Mal. Cour., 5, 777.

Gibson, A. G. (1907). Lancet, 2, 1380.

Hirschfeider, A. D. (1907). Johns Hopk. Hosp. Bull., 18, 265.

Melik-Gülnusarian, E. A. (1932). Z. KreislForsch., 24, 433.

Obrastzow, W. P.(1905). Z. klin. Med., 56, 70.

and Gubergritz, M. M. (1919). Quoted by Melik-Gülnasarian (1932).

Potain, $\underset{\text { (1867). }}{\text { C Bull. Soc. Séd Hôp. Paris, 3, } 138 .}$

(1867). Bull. Soc. méd

(1880). Ibid., 12, 137. Hôp., 53, 529.

(1894). Clinique médicale de la Charité : Leçons et Mémoires. Masson, Paris.

(1900). Semaine méd., 20, 175

Steinberg, L. D. (1925). Z. Kinderheilk., 40, 620

Thayer, W. S. (1908). Trans. Ass. Amer. Phys., 23, 326

(1909). Arch. intern. Med., 4, 297.

White, P. D. (1928). Ibid., 41, 1.

(1944). Heart Disease, Macmillan.

Wolferth, C. C., and Margolies, A. (1933). Amer. Heart J., 8, 441.

\section{SEVERE ERYTHEMA MULTIFORME}

\author{
BY
}

\section{B. SNEDDON, M.B., Ch.B., M.R.C.P.}

\section{Honorary Physician to Skin Department, Royal Sheffield} Infirmary and Hospital

Numerous papers describing cases of severe erythema multiforme have appeared in American and Continental dermatological literature. There is, however, a scarcity of reports in British journals, the most recent being that of Low and Davies in 1938. . The comparative frequency of the syndrome is considered justification for the following case reports. In the early stages the disease may present itself as a sudden pyrexial illness associated with stomatitis which simulates diphtheria, Vincent's angina, glandular fever, or one of the exanthemata. Therefore a knowledge of the condition is of value to clinicians who are not pure dermatologists.

According to Beaudonnet (1894) the first description of the condition is said to have been by Alibert and Bazin in 1822. It has been rediscovered on several occasions, and each discoverer has coined a new name. Thus Fiessinger and Rendu (1917) called it "ectodermose erosive pluriorificielle," Stevens and Johnson (1922) "a new eruptive fever with stomatitis and ophthalmia," and Baader (1925) " dermatostomatitis." Lever (1944) surveyed the literature very fully and concluded that, on the evidence presented, the cases described under the above three names were all the same condition-erythema multiforme. He also suggested that some of the published cases of acute pemphigus and some instances of human footand-mouth disease in which laboratory confirmation of the diagnosis was not obtained were also examples of severe erythema multiforme.

I have seen and treated nine cases of severe erythema multiforme in the last four years-one in a child, seven in young adults, and one in a woman of 55 . It is therefore not a great rarity. The following cases are described as illustrative of the disease.

\section{Case 1}

A single woman aged 25 was seen on Sept. 3, 1946, complaining of severe pruritus ani and vulvae of one month's duration. She had no past. history of skin or allergic disease and her only previous illnesses had been nephritis when 6 , and anaemia, which had responded to iron therapy, when 21 .

On examination the skin around the anus and vulva was sodden and excoriated, but no underlying cause was found. She was treated with a kaolin lotion containing $1 \%$ phenol and a dose of $100 r$ of superficial $x$ rays to the affected areas. Phenobarbitone was given, $1 / 2 \mathrm{gr}$. (32 mg.) in the morning and 1 gr. $(65 \mathrm{mg}$.) at night. The symptoms improved on this regime, and when she was seen again on Sept. 17 the phenobarbitone was discontinued and another dose of $x$ rays given. On Sept. 23 the pruritus vulvae became more intense and the vagina became sore, causing discomfort on micturition. The following day ulcers appeared in the mouth and on the lips; she became feverish and felt ill with headache, backache, and malaise. Towards the end of the day a generalized eruption appeared on the trunk and limbs. On Sept. 25 her temperature rose to $104^{\circ}$ F. $\left(40^{\circ}\right.$ C. $)$, the eruption became worse, and a bilateral conjunctivitis started. She was therefore admitted to hospital.

On admission her temperature was $102^{\circ} \mathrm{F} .\left(38.9^{\circ} \mathrm{C}\right.$. $)$, pulse 88 , respirations 20 . Her eyelids were stuck together by a glutinous mucopurulent exudate, her face was swollen and unrecognizable, the whole of the inside of her mouth was covered with a grey sodden pseuc'o-membrane, and her lips were swollen and crusted. A diffuse eruption consisting of purplish red papules 0.5 to $1 \mathrm{~cm}$. in diameter was present on the trunk, but on the limbs it was frankly bullous. The bullae arose in the centre of papules, and none appeared on normal skin. Both palms and soles showed erythematous macules, with a few flaccid bullae on the tips of fingers and toes. The face and scalp were not involved. Nicolsky's sign was not present. There was a profuse vaginal discharge and the vulva and vagina showed shallow ulceration. The anus appeared normal. There was no enlargement of lymphatic glands, liver, or spleen, and no abnormality was found in the cardiovascular, respiratory, and nervous systems.

During the next four days her fever continued and a number of the bullous lesions became secondarily infected. Micturition was impossible without the help of warm vaginal douches. On the fifth day after admission her temperature returned to normal, her general condition improved, and the necrotic mucous membrane in the mouth began to separate. A few fresh bullae were still appearing on her legs, but most of the papular eruption on the trunk had faded and the conjunctivitis showed improvement. During the next week all the lesions improved rapidly, the ulceration of the lips being the most troublesome and the slowest to teal. She could now pass water without difficulty. On Nov. 15, 21 days after admission, her mouth and lips were normal and all that remained of the skin eruption was faint erythematous staining and slight desquamation. The conjunctivitis had recovered completely and she had no recurrence of the pruritus ani and vulvae.

On Oct. 26 the leucocytes numbered 11,000 per c.mm. (polymorphonuclears, $72 \%$; lymphocytes, $22 \%$; monocytes, $2 \%$; eosinophils, $4 \%$ ). Blood culture was sterile. Culture from vaginal discharge gave a growth of $\beta$ haemolytic streptococcus and diphtheroids. Culture from the mouth gave a growth of $\beta$ haemolytic streptococcus and Staphylococcus albus (coagulase-positive). On Oct. 29 the leucocytes numbered 10,200 ; on Nov. 5 the haemoglobin was $72 \%$, and the erythrocytes $3,640,000$ per c.mm. A skiagram of the chest revealed no abnormality. The Mantoux test was $1: 10,000$ negative, $1: 1,000$ positive. Treatment consisted of local symptomatic measures, and after the result of the bacteriological examination $1,200,000$ units of penicillin were given intramuscularly from Oct. 28 to Nov. 2.

\section{Case 2}

A boy aged 9 was admitted to an isolation hospital on Oct. 14, 1946. For a month prior to admission he had been run down and "nervy." Four days before admission his eyes became inflamed and swollen, and on the same day he bit his lower lip. The lesion on the lip became sore, and ulceration spread to the inside of his mouth. Two days later a few pink spots appeared on his upper chest, his eyes became more inflamed, and blisters were seen insidę his mouth. His previous history contained no relevant facts.

On admission his temperature was $100^{\circ}$ F. $\left(37.8^{\circ}\right.$ C. $)$ and both eyes were closed by swelling and sticky mucopurulent 